\title{
Dual-Beam Interferometry for Ocean Surface Current Vector Mapping
}

\author{
Stephen J. Frasier, Member, IEEE, and Adriano J. Camps, Associate Member, IEEE
}

\begin{abstract}
The recent use of along-track interferometry (ATI) in synthetic aperture radar (SAR) has shown promise for synoptic measurement of ocean surface currents. ATI-SARs have been used to estimate wave fields, currents, and current features. This paper describes and analyzes a dual-beam along-track interferometer to provide spatially resolved vector surface velocity estimates with a single pass of an aircraft. The design employs a pair of interferometer beams, one squinted forward and one squinted aft. Each interferometric phase is sensitive to the component of surface Doppler velocity in the direction of the beam. Therefore, a proper combination of these measurements provides a vector surface velocity estimate in one pass of the aircraft. We find that precise measurements dictate widely spaced beams and that the spatial resolution for the squinted SAR is essentially identical to the sidelooking case. Practical instrument design issues are discussed, and an airborne system currently in development is described. Through computer simulation, we observe the azimuthal displacement of interferometric phases by moving surfaces identical to those of conventional SAR and find that such displacement can bias the estimated surface velocity.
\end{abstract}

Index Terms-Along-track, interferometry, ocean surface currents.

\section{INTRODUCTION}

$\mathbf{T}$ HE USE of along-track interferometric SAR (ATI-SAR) has shown promise for synoptic measurement of ocean surface currents. Goldstein et al. [1] reported early comparisons of ATI-SAR and surface drifters. Since then, interferometric SARs have also been used to estimate wave fields [2] and current features [3], [4]. Recent comparisons between ATI-SAR and shore-based HF radar current mapping systems have shown reasonable agreement when appropriate corrections are applied [5]. To date, however, interferometric measurements of surface currents have employed sidelooking SARs that implement a single beam. As such, only one radial component of Doppler surface velocity is obtained in any one pass of the aircraft. Two passes, ideally orthogonal, are required to obtain a vector measurement, during which time the current field is assumed to be constant. Though usually valid for large-scale current features, this assumption does require that the passes be made as close together as possible. Furthermore, vector estimates can only be made

Manuscript received September 13, 1999; revised July 7, 2000. This work was supported by the Office of Naval Research (Remote Sensing) Grant N00014-98-1-0612 and Fellowship PR98 0046131105 of the Spanish Ministry of Education and Culture. This work was performed while A. Camps was on sabbatical at the University of Massachusetts, Amherst.

S. J. Frasier is with the Department of Electrical and Computer Engineering, University of Massachusetts, Amherst, MA 01003 USA.

A. J. Camps is with the Universitat Politecnica de Catalunya, 08034, Barcelona, Spain.

Publisher Item Identifier S 0196-2892(01)01170-6.

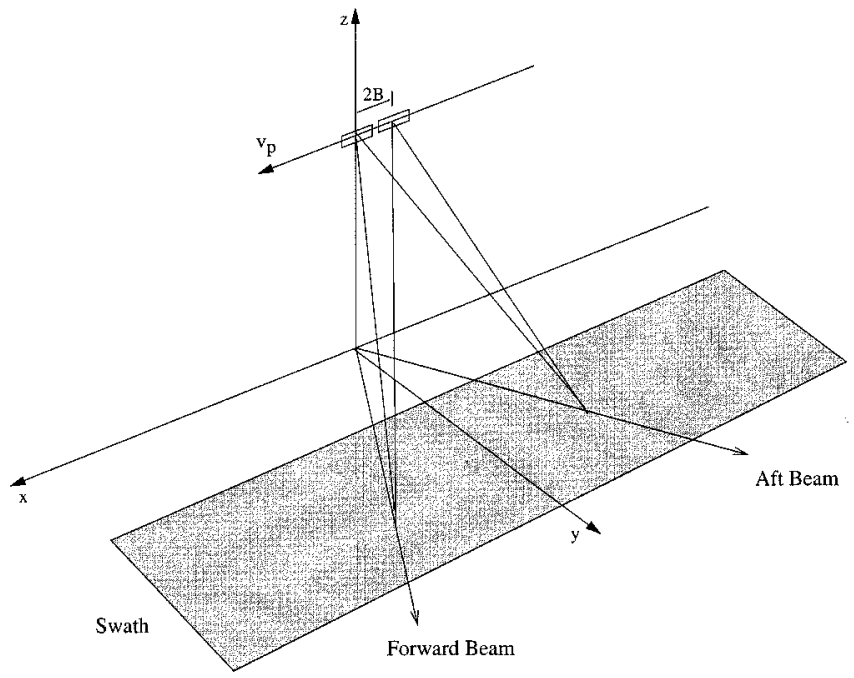

Fig. 1. Dual-beam interferometer system geometry.

over the limited area where SAR images overlap, making long distance strip mapping impractical.

In this paper, we discuss a technique for estimating surface current vectors using a modest extension to the ATI-SAR, originally suggested by Rodriguez [6], which we term a dual-beam interferometer (DBI). The basic concept of the DBI is shown in Fig. 1, in which a coherent airborne radar employs an alongtrack pair of dual-beam antennas, each antenna producing a forward and an aft beam. By virtue of the forward motion of the aircraft, echoes from the forward beams are shifted up in frequency, while echoes from the aft beams are shifted down in frequency. Spatially co-registered echoes from the beams of the aft antenna are displaced in time from those of the fore antenna by the time required to traverse the antenna baseline distance $2 B$. Radar echoes from the two forward beams and from the two aft beams are each cross-correlated to yield a pair of interferograms. The phase of each interferogram provides one line-of-sight component to the Doppler surface velocity. Vector estimates are then obtained by appropriate combination of the separate interferograms.

This paper is organized in four sections. Section II reviews the basic principle of along-track interferometric (ATI) SAR. The response of a squinted ATI-SAR, including platform velocity/attitude errors is derived and discussed. It includes a treatment of ocean surface coherence properties at microwave frequencies, effects of SNR, and the impact of motion errors. A description of an airborne DBI system currently in development is provided in Section III. Finally, Section IV describes computer simulation 


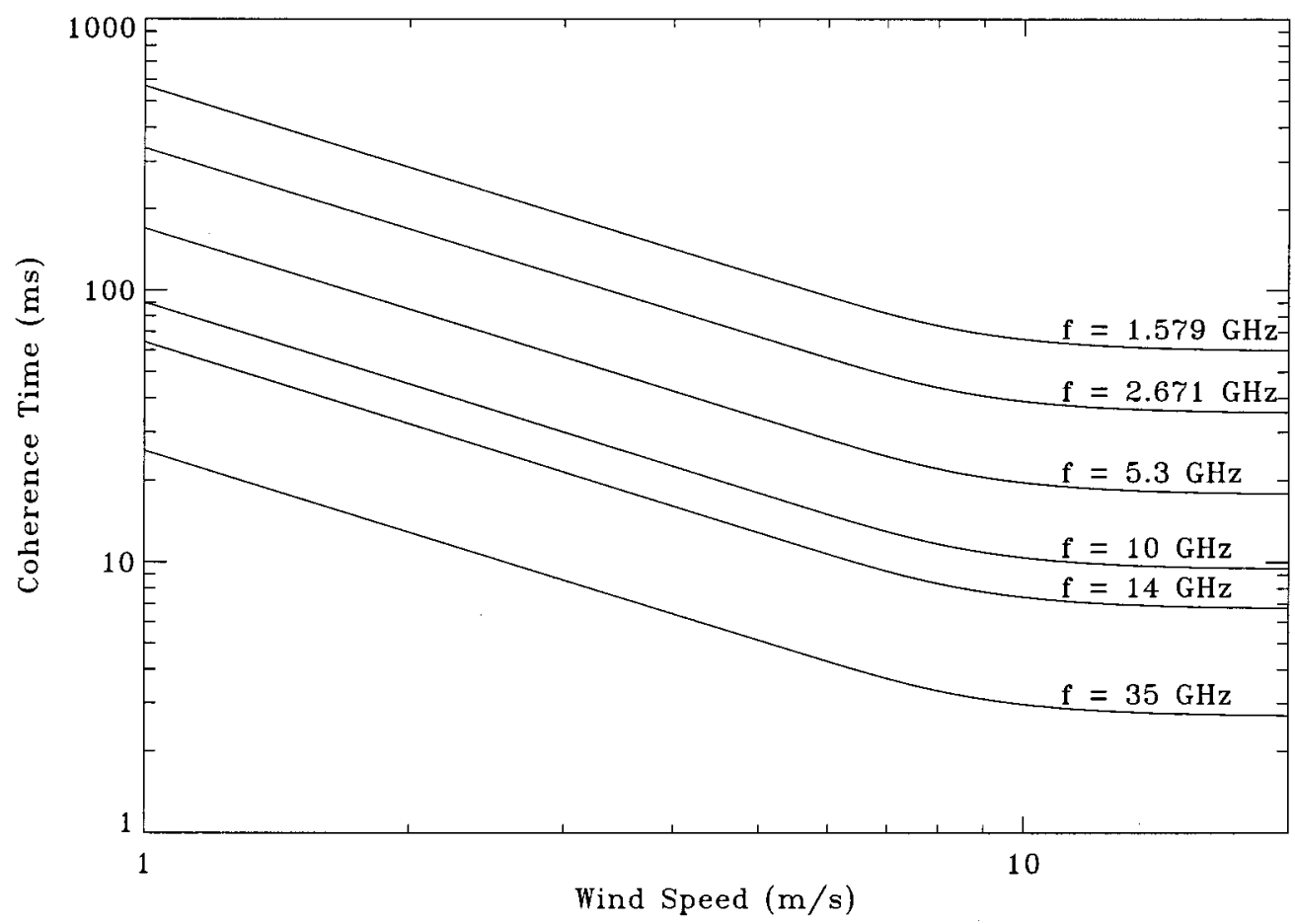

Fig. 2. Predicted coherence time versus wind speed at six different frequencies for a spatial resolution of $30 \mathrm{~m}$.

results of a DBI system. Intrinsic measurement limitations for large range and/or current velocities are observed, as well as the way to mitigate them.

\section{ALONG-TRACK INTERFEROMETRY}

The basic concept of along-track interferometry is to consider two phase coherent radars deployed along the side of an aircraft. When the radar echoes produced by the fore and aft antennas, $V_{1}(t)$ and $V_{2}(t+\tau)$, are spatially co-registered, the aft image lags the fore image by the time required for the aft antenna to advance to the (prior) location of the fore antenna: $\tau=2 B / v_{p}$, where $2 B$ is the baseline distance between the antennas, and $v_{p}$ is the aircraft's horizontal velocity. Thus, the two antennas provide two observations of the same surface from the same location at slightly different times. Any change in phase observed between these looks is due to the mean Doppler velocity of the surface scatterers $v_{0}$, which is obtained from the cross correlation

$$
v_{0}=\frac{\lambda}{2} \frac{\arg \left\langle V_{1}(t) V_{2}^{*}(t+\tau)\right\rangle}{2 \pi \tau}
$$

where $\lambda$ is the electromagnetic wavelength. Surface current is then inferred from this velocity measurement. The unambiguous range of values for the phase is $[-\pi, \pi]$, which yields an unambiguous Doppler velocity interval of $[-\lambda / 4 \tau, \lambda / 4 \tau]$. In practice, one of the antennas is often transmit-receive, while the other is receive-only. This halves the effective baseline between the antennas, as the radar comprised of both the fore and aft antennas has a phase center located midway between the physical antennas.

\section{A. Ocean Surface Coherence Properties}

Critical to the design of an interferometer is the choice of the baseline. A value too small leads to observations very closely spaced in time, making Doppler estimates sensitive to noise. A value too large leads to decorrelation of the backscatter between observations, yielding no information on velocity. The choice of interferometric baseline hinges on sufficient correlation between the two successive looks at the surface. For this, one needs an estimate of the coherence time $\tau_{s}$ of scattering from the ocean surface. This is known to be a function of both illuminated surface area and of the "lifetimes" of the Bragg-resonant scattering facets. Plant et al. [7] have shown that lifetime effects are important only for small illuminated areas. For larger illuminated areas (>several m), the root mean square (RMS) velocity spread within the illuminated area dictates the Doppler bandwidth and hence the coherence time. The RMS velocity spread is essentially the range of observed orbital velocities of the larger-scale gravity waves whose RMS value is a function of sea-state. Values of order $0.50 \mathrm{~m} \cdot \mathrm{s}^{-1}$ (Plant's observation) to $2 \mathrm{~m} \cdot \mathrm{s}^{-1}$ are typical.

Given a surface displacement spectrum $S_{\eta}(\mathbf{K})$, the coherence time can be estimated from the radial component of the RMS orbital velocity (see, e.g., [8])

$$
\begin{aligned}
\tau_{s} & =\sqrt{2} \frac{1}{2 k \sigma_{v}}, \\
\sigma_{v}^{2} & =\int_{K_{\min }}^{\infty}|g|^{2} \Omega_{K}^{2} S_{\eta}(\mathbf{K}) d \mathbf{K}, \\
|g|^{2} & =\cos ^{2} \theta+\sin ^{2} \theta \sin ^{2} \phi
\end{aligned}
$$


where

$\theta \quad$ incidence angle;

$\phi \quad$ wave propagation angle relative to look direction;

$K_{\min }$ spatial resolution;

$\Omega \quad$ ocean wave radian frequency.

Fig. 2 shows estimated coherence times versus wind speed for six different frequencies given a spatial resolution of $30 \mathrm{~m}$ and a Pierson-Moskowitz-type wave spectrum [9], [10]. For this case, it is assumed that the radar beam is aligned with the waves, and the coherence time is given approximately by

$$
\tau_{s} \approx 3 \frac{\lambda}{u} \operatorname{erf}^{-1 / 2}\left(2.7 \frac{\rho}{u^{2}}\right)
$$

where $\rho$ is the spatial resolution, and $u$ is the wind speed. For low-to-moderate resolutions and wind speeds, $\tau_{s} \approx 3 \lambda / u$. These values are consistent with reported coherence times of $3,7,10,35$, and $58 \mathrm{~ms}$ at $35,14,10,2.671$, and 1.579 $\mathrm{GHz}$, respectively [11]. Coherence times may be expected to increase somewhat when the radar is oriented cross-wave as line of sight velocity variances will decrease. However, this will depend upon the particular directional spectrum. Based on (3), the coherence time achieves its minimum value for wind speeds above about $10 \mathrm{~ms}^{-1}$. It is important to note that winds of approximately $2-4 \mathrm{~ms}^{-1}$ are typically required to generate sufficient small scale roughness to produce a radar echo. Thus, the dynamic range of coherence times predicted by this model is not particularly large.

For scattering from the ocean surface, the spatial resolution that can be achieved using synthetic aperture techniques is dictated not by the available integration time due to the antenna beamwidths but by surface motions and by the coherence time of the surface scatterers. Typical expressions for azimuth resolution have the form [12], [13]

$$
\rho_{a}=\sqrt{\left(\frac{\lambda R}{2 v_{p} T_{i}}\right)^{2}+\left(\frac{\lambda R}{2 v_{p} \tau_{s}}\right)^{2}+\left(\frac{\pi T_{i} R}{2 v_{p}} a_{r}\right)^{2}}
$$

where

$T_{i} \quad$ integration time;

$v_{p} \quad$ platform velocity;

$\tau_{s} \quad$ coherence time;

$a_{r} \quad$ surface acceleration.

The first term is the theoretical SAR resolution for stationary coherent targets where the maximum available integration time is dictated by the antenna beamwidth, the range, and the platform velocity. The second term accounts for the finite coherence time of the surface scatterers, while the third term represents defocusing due to surface orbital accelerations. The first two terms are often combined to yield an effective integration time $T_{a}$, given by

$$
\frac{1}{T_{a}^{2}}=\frac{1}{T_{i}^{2}}+\frac{1}{\tau_{s}^{2}} .
$$

Thus, the effective integration time is always less than either the coherence time or the actual integration time used. For typical aircraft speeds, this limit on integration time often determines the spatial resolution achievable. Spatial resolution is optimized by setting the integration time to a small multiple of the coherence time, such that the term in (4) involving coherence time dominates the expression. ${ }^{1}$

\section{B. Surface Current Estimation}

Before proceeding further, a note of caution is in order. The measurement produced by the ATI-SAR is not the true surface current but a measure of the surface Doppler velocity, which is a power-weighted sum of the line-of-sight velocities within a given resolution cell of the radar. Contributors to the Doppler velocity include the line-of-sight components of 1) the phase velocities of radially travelling Bragg-resonant surface waves that are primarily responsible for the microwave echo; 2) the orbital velocities of long surface waves; and 3) the surface current, which includes wind-induced and wave-induced drift components.

To obtain an estimate of the surface current, a proper accounting for the effects of items 1 and 2 must be made. Accounting for Bragg-resonant phase velocity, $c_{p}$, requires knowledge of the directional spreading of these waves on the ocean surface. Because the Bragg scattering mechanism "selects" only radially travelling waves (both advancing and receding), the net Doppler velocity observed by the radar due to the Bragg-resonant waves alone lies between $\pm c_{p}$ depending upon their directional distribution. This is generally not an issue when looking directly upwind or downwind, but needs to be considered when looking obliquely or crosswind. In this case both advancing and receding Bragg-resonant waves can contribute. Thompson and Jensen [3] demonstrated that significant errors can occur if care is not taken. Directional spreading is typically modeled by

$$
G\left(\theta_{w}\right)=\cos ^{2 n}\left(\frac{\theta_{w}}{2}\right)
$$

where $\theta_{w}$ is the wind direction relative to the radar boresight and $n$ is a spreading factor, typically 10 near the spectral peak and 2-5 in the intermediate range of the spectrum [14], [15]. Though the degree of angular spreading is sea state dependent, Poulter [16] found $n \approx 2.5$ for Bragg resonant $5 \mathrm{~cm}$ waves, while Moller [17] found a narrower distribution, $n \approx 4$, for Bragg resonant $1.5 \mathrm{~cm}$ waves. These observations are consistent with evidence that the angular spreading of short capillary waves is confined by the influence of larger gravity waves [18]. Clearly, however, further investigations are needed.

Accounting for orbital velocity effects requires knowledge of the coupling between radar echo power and Doppler. These are known to be correlated and are described by the radar modulation transfer function (MTF) [19], relating backscattered power to wave slope (or orbital velocity). Chapman [11], and Moller [17] illustrate the influence of MTF on phase difference measurements typical of ATI-SARs.

From this introductory discussion, it is evident that conversion of microwave Doppler measurements to surface currents

\footnotetext{
${ }^{1}$ In the absence of accelerations, $\rho_{a}$ is minimized when $T_{i}$ is maximized. However, the third term may become dominant if $T_{i} \geq 2 /\left(k \tau_{s} a_{r}\right)$. Thus, spatial resolution is optimized when $T_{i}$ is larger than but of similar order to $\tau_{\mathcal{s}}$.
} 
is itself a nontrivial task with several potential sources of error whose dependence upon environmental parameters is a topic of research. Despite these sources of uncertainty, reasonable comparisons have been made between ATI-SAR and other techniques. In their study, Graber et al. [5] employed two different methods to account for these effects: one using in situ measurements (buoys) as tie-points, the other using a scattering model to predict the Doppler bias in the absence of current.

In the present study, we do not attempt to incorporate these sources of error into our analysis. Our attention is instead restricted to instrumental and platform induced errors. While we have considered the presence of surface waves on the ocean to obtain expressions for surface coherence properties and spatial resolution, we limit our analysis to a constant current field as a simple test case for the response of a DBI. Thus, expressions obtained should be interpreted as a lower bound on the accuracy of surface current measurements.

\section{DBI Point Source Response}

Following a procedure similar that of [13], [20], [21], and considering short integration times, the response of a squinted ATI-SAR is derived in Appendix A, including the effects of platform attitude and velocity errors. According to (45) of the Appendix, the normalized along-track response to a point source located at $R=\left(x_{0}, y_{0}, 0\right)$ is a Gaussian function given by

$$
\begin{aligned}
I\left(x, y_{0}\right) & =\exp \left(-\pi^{2}\left(x-x_{\max }\right)^{2} / \rho_{a}^{2}\right) \\
x_{\max } & =\frac{\mathbf{R} \cdot \mathbf{v}}{v_{p}}=x_{0}+\frac{R}{v_{p}} u_{r} \\
\rho_{a} & =\lambda R / 2 T_{a} v_{p}
\end{aligned}
$$

where

R vector from the midpoint of the along-track baseline to the surface;

$\mathbf{v}=\mathbf{v}_{\mathbf{p}}-\mathbf{v}_{\mathbf{0}}$ relative velocity of the surface point $\mathbf{v}_{\mathbf{0}}$, with respect to the platform velocity $\mathbf{v}_{\mathbf{p}}$;

$u_{r} \quad$ radial velocity of the imaged source.

Thus, the center of the image is displaced from the true position $x_{0}$, as expected. The phase at $x_{\max }$ is

$$
\Phi\left(x_{\max }\right)=-\frac{2 k B}{R}\left[\frac{\mathbf{R} \cdot \mathbf{v}}{v_{p}}-\frac{\mathbf{R} \cdot \mathbf{B}}{B}\right]
$$

from which one estimates radial velocity in the direction of the squinted beam. In the case of a horizontal platform velocity and a horizontal baseline, (8) reduces to the expected result

$$
\Phi\left(x_{\max }\right)=\frac{2 k B}{v_{p}} u_{r}=k \tau u_{r}
$$

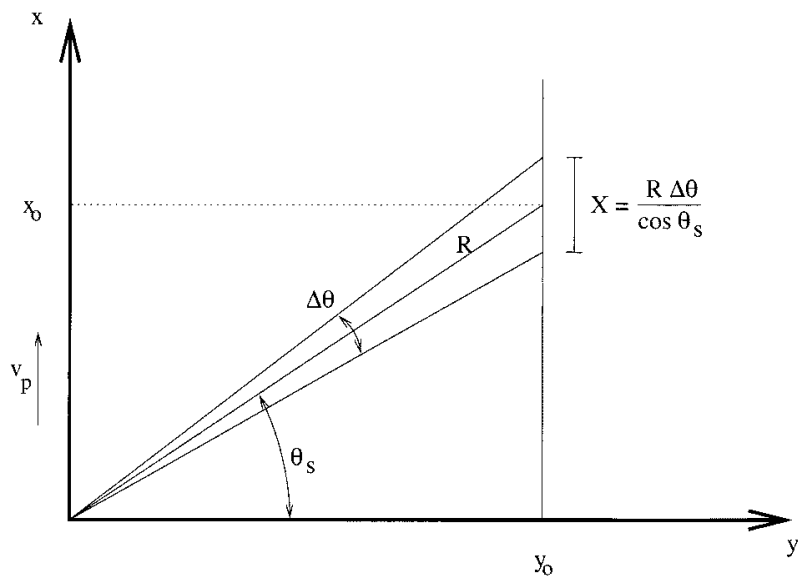

Fig. 3. Geometry used to compute the number of available looks.

where $\tau$ is the interferometric delay $2 B / v_{p}$. To estimate $u_{r}$, one divides the measured phase by $k \hat{\tau}$, where $\hat{\tau}$ is obtained from an independent estimate of the platform velocity, $\hat{v}_{p}$. That is

$$
\hat{u}_{r}=\frac{\hat{v}_{p}}{2 k B} \Phi .
$$

Thus, $\hat{u}_{r}$ (the estimate of $u_{r}$ ) depends upon accurate knowledge of the platform velocity. Given the estimated radial velocities from the forward and aft beams, the along-track and cross-track components of the horizontal surface velocity are obtained using simple geometry

$$
\hat{v}_{0 x}=\frac{\hat{u}_{r}^{+}-\hat{u}_{r}^{-}}{2 \sin \theta_{s} \sin \theta_{i}}, \quad \hat{v}_{0 y}=\frac{\hat{u}_{r}^{+}+\hat{u}_{r}^{-}}{2 \cos \theta_{s} \sin \theta_{i}}
$$

where the plus and minus superscripts correspond to forward and aft beams, respectively. The variances of these current estimates are

$$
\sigma_{v_{0 x}}^{2}=\frac{\sigma_{u_{r}^{+}}^{2}+\sigma_{u_{r}^{-}}^{2}}{\left(2 \sin \theta_{s} \sin \theta_{i}\right)^{2}}, \quad \sigma_{v_{0 y}}^{2}=\frac{\sigma_{u_{r}^{+}}^{2}+\sigma_{u_{r}^{-}}^{2}}{\left(2 \cos \theta_{s} \sin \theta_{i}\right)^{2}}
$$

and the total velocity variance is the sum of these, which can be related to the measured phase uncertainty using (8), yielding

$$
\sigma_{v_{0}}^{2}=\frac{\sigma_{\Phi^{+}}^{2}+\sigma_{\Phi^{-}}^{2}}{\left(k \tau \sin 2 \theta_{s} \sin \theta_{i}\right)^{2}}
$$

\section{Signal-to-Noise Evaluation}

For interferometry, the primary concern with SNR is its effect on phase estimates, and hence, radial velocity estimates in the 


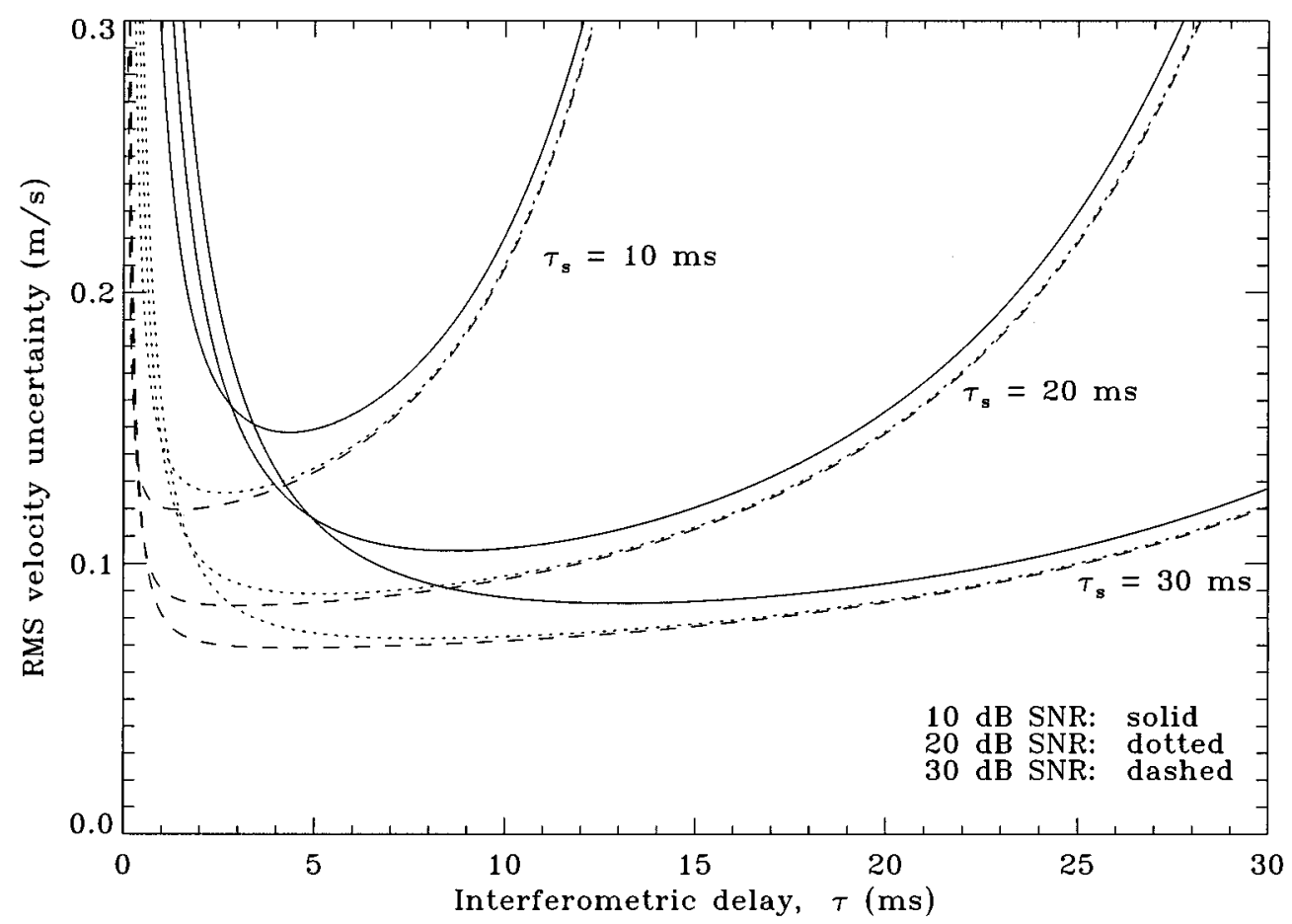

Fig. 4. Velocity uncertainty versus interferometric time lag $\tau$ for coherence times of 10, 20, and $30 \mathrm{~ms}$ and for SNR of 10, 20, and 30 dB.

fore and aft directions. One can express the standard deviation of the interferometric phase estimate as [22]-[24]

$$
\sigma_{\Phi}=\frac{\sqrt{\left(1+\frac{1}{\mathrm{SNR}}\right)^{2}-\rho^{2}(\tau)}}{\sqrt{2 N} \rho(\tau)}
$$

where $\rho(\tau)=\exp \left(-\tau^{2} / \tau_{s}^{2}\right)$ is the correlation coefficient between observations separated by $\operatorname{lag} \tau$, and $N$ is the number of independent looks at a given resolution cell that are averaged.

The number of independent looks available can be estimated from the from the illumination time $T_{0}$, divided by the coherent integration time $T_{i} . N$ depends on the cross-track distance $y_{0}$, and also on the squint angle $\theta_{s}$ according to

$$
N=\frac{T_{0}}{T_{i}} \approx \frac{R \Delta \theta}{T_{i} v_{p} \cos \theta_{s}}
$$

where $\Delta \theta$ is the beamwidth of the squinted antenna (Fig. 3). Additional looks are also available by spatial averaging within the image at the expense of spatial resolution.

Finally, substituting (14) and (15) in (13), the variance of the surface current magnitude is given approximately by

$$
\begin{aligned}
\sigma_{\imath 0}^{2} \approx & \frac{v_{p}}{k^{2} R \Delta \theta} \frac{\cos \theta_{s}}{\sin ^{2} 2 \theta_{s} \sin ^{2} \theta_{i}} \frac{T_{i}}{\tau^{2} \rho^{2}(\tau)} \\
& \cdot\left[1-\rho^{2}(\tau)+\frac{1}{2}\left(\frac{1}{\mathrm{SNR}^{+}}+\frac{1}{\mathrm{SNR}^{-}}\right)\right]
\end{aligned}
$$

Note that (16) has three terms within the brackets. The first is the variance due to decorrelation, while the second and third represent the SNR observed in the fore and aft beams. These values are dependent on the sea state, wind speed, and wind direction. This expression provides an optimistic estimate of the velocity uncertainty because it ignores any spatial and temporal variability of the surface velocity due to the long surface waves that may be resolved by the radar. That is, it assumes an essentially flat sea. It does, however, set a lower bound on the precision of mean velocity estimates due to decorrelation and finite SNR.

Fig. 4 shows the estimated velocity standard deviation as a function of interferometric time lag $\sigma_{v 0}(\tau)$ for $T_{i}=3 \tau_{s}$. That is, for coherent integration time larger than, but of the same order as, surface coherence time. Here we have chosen the parameters $\lambda=2 \pi / k=6 \mathrm{~cm}$ (C-band), $v_{p}=50 \mathrm{~ms}^{-1}, \theta_{i}=\theta_{s}=45^{\circ}$, and $R \approx 3 \mathrm{~km}$. These are consistent with operation from small aircraft.

The optimum value of $\tau$ depends on both the SNR and the coherence time. The figure illustrates two important points. First, the estimate is nearly optimum for SNR of $20 \mathrm{~dB}$ [22]. There is little reason to design for much better SNR than this. Second, for a given SNR and coherence time, there is an optimum choice of $\tau$ providing the best tradeoff between the competing effects of noise, which dominates for small $\tau$, and decorrelation, which dominates for large $\tau$ [25]. For SNR about 10 and $\tau_{s}=10-20$ $\mathrm{ms}$, a minimum occurs for $\tau$ between 5 and $10 \mathrm{~ms}$.

From (16), for a given SNR and correlation coefficient, the optimum squint angle that minimizes the variance of the total estimated velocity is given by the minimum of the geometric term $\cos \theta_{s} / \sin ^{2} 2 \theta_{s}$, which actually occurs at $\theta_{s}=54.7^{\circ}$. This yields an angle between fore and aft looks of about $110^{\circ}$. Intu- 


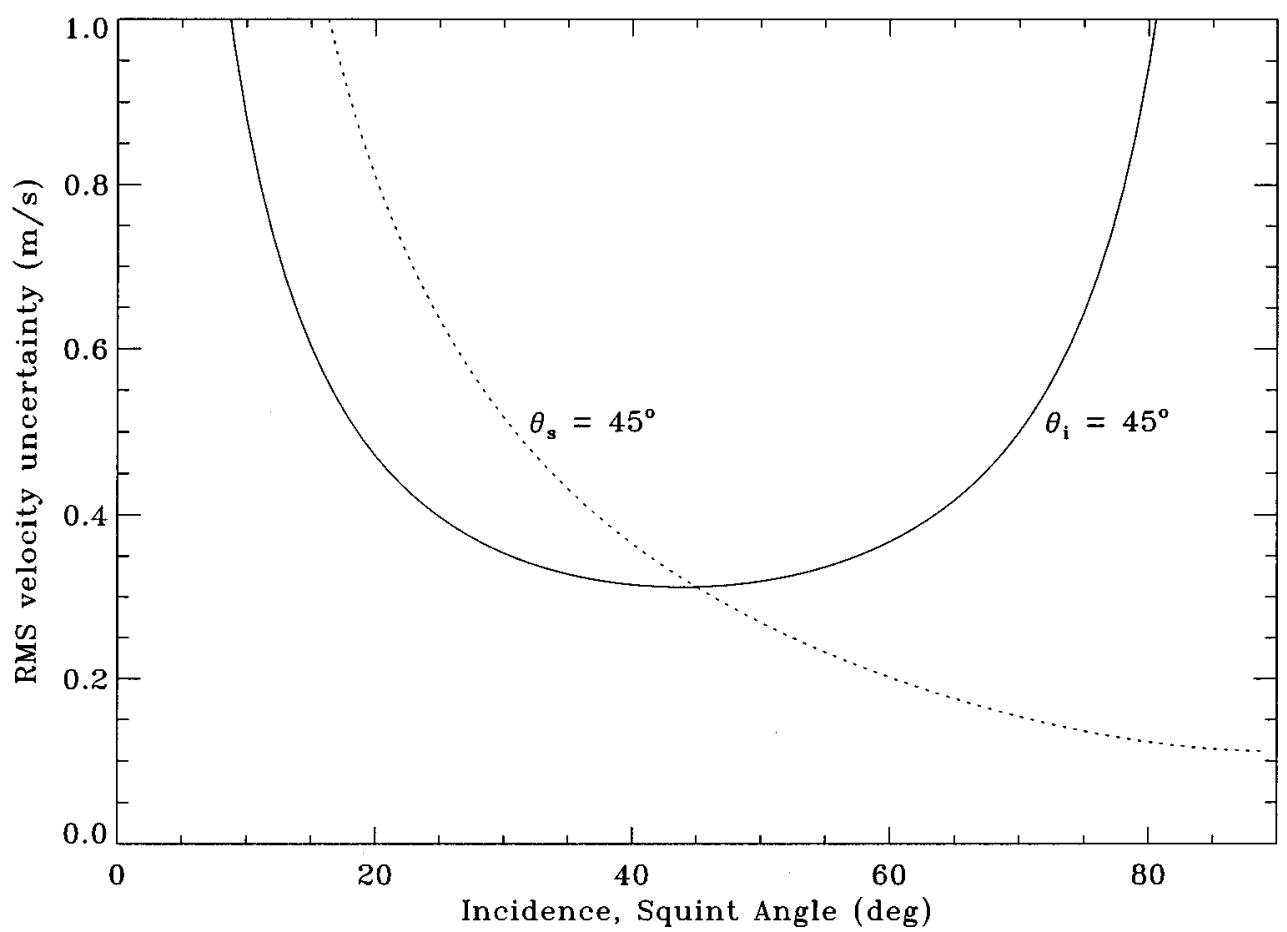

Fig. 5. Single-look velocity uncertainty versus squint and incidence angles given platform uncertainties $\sigma_{v_{p}}=0.1 \mathrm{~m} / \mathrm{s}, \sigma_{v_{z}}=0.2 \mathrm{~m} / \mathrm{s}$, and $\sigma_{\beta}=\sigma_{\gamma}=1 \mathrm{mrad}$. Solid line: squint angle dependence at $\theta_{i}=45^{\circ}$. Dotted line: incidence angle dependence at $\theta_{s}=45^{\circ}$.

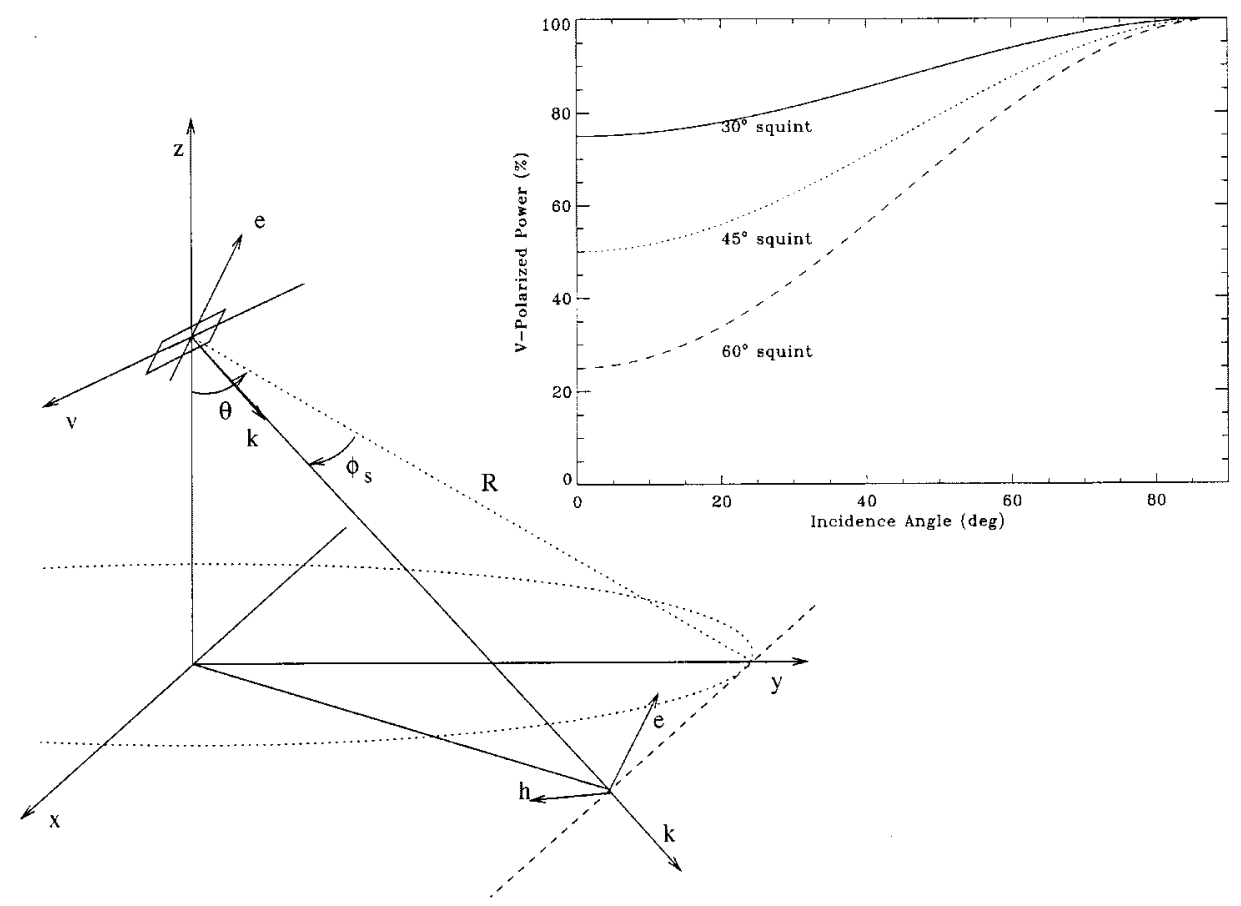

Fig. 6. Geometry detailing squinted versus rotated antenna boresights. Inset: Percentage of vertically polarized power versus incidence angle for a squinted sidelooking antenna.

itively, $90^{\circ}$ between fore and aft looks would seem to be optimum. This is, in fact, the angle that yields equal uncertainties in cross-track and along-track velocities assuming equal SNRs in the fore and aft directions. In any case, this dependence on squint angle is weak over a range of moderate squint angles. Therefore, the selection of the squint angle in terms of variance of the estimated phase due to SNR and decorrelation is not a critical issue, provided it is at least $20^{\circ}$ or so.

\section{E. Velocity and Attitude Tolerances}

The surface velocity vector is obtained by combining the interferometric phases measured by the fore and aft beams, (11). 

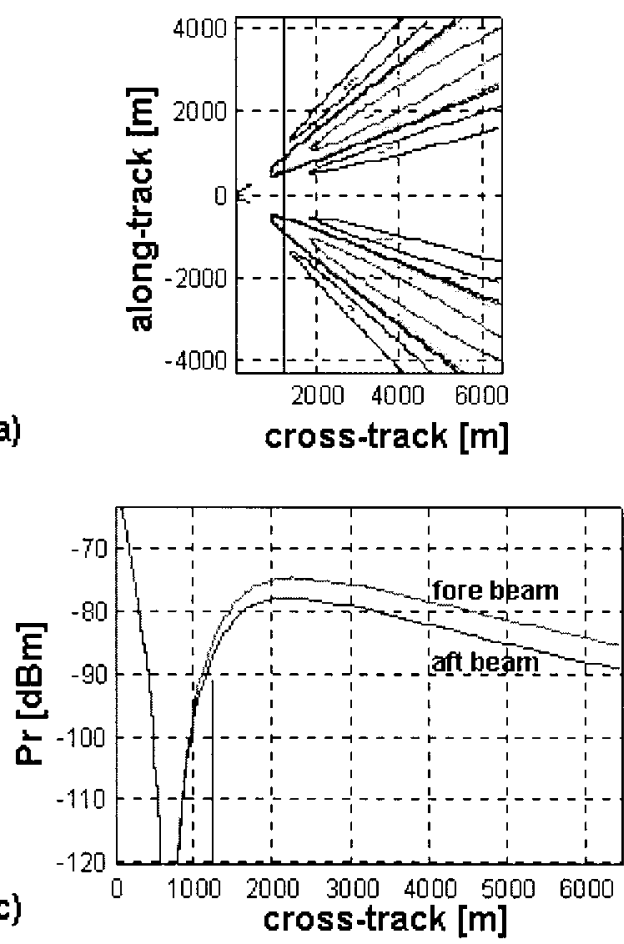
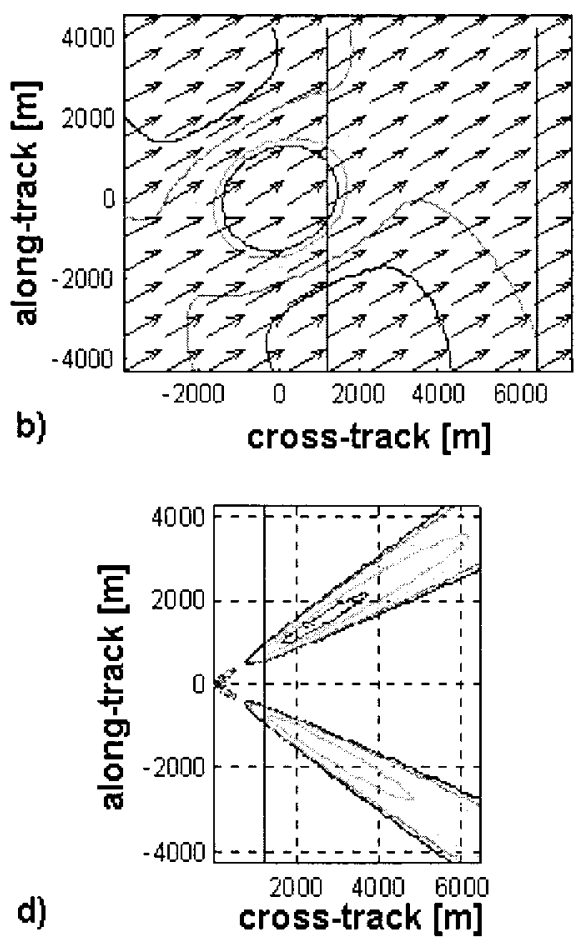

Fig. 7. (a) Antenna pattern footprint contours of fore and aft beams ( $-3 \mathrm{~dB},-10 \mathrm{~dB},-15 \mathrm{~dB} \ldots$ ). (b) Constant $5 \mathrm{~m} / \mathrm{s}$ wind field overlaid on $\sigma^{\circ}$ contours ( -15 $\mathrm{dB},-17.5 \mathrm{~dB},-20 \mathrm{~dB}$...) obtained using the CMOD4 model function. (c) Received power along the fore and aft beams versus ground range. (d) Received power contour plot $(-3 \mathrm{~dB},-10 \mathrm{~dB},-20 \mathrm{~dB} \ldots)$.

If the integration time is of the order of the coherence time such that the acceleration error terms can be neglected, the radial velocities in the fore and aft directions can be written

$$
\begin{aligned}
u_{r}^{ \pm}= & \pm \sin \theta_{i} \sin \theta_{s}\left[v_{0 x}-\Delta v_{p}-v_{p}(1-\cos \beta \cos \gamma)\right] \\
& +\sin \theta_{i} \cos \theta_{s}\left(v_{0 y}+v_{p} \cos \beta \sin \gamma\right) \\
& \left.+\cos \theta_{i}\left[\left(\Delta v_{z}-v_{0 z}\right)+v_{p} \sin \beta\right)\right]
\end{aligned}
$$

where

$\beta$ and $\gamma$ respective pitch and yaw angles of the baseline;

$\Delta v_{p} \quad$ error in estimated platform horizontal velocity;

$\Delta v_{z} \quad$ vertical velocity of the platform.

Inserting (17) in (11) yields the following forms for the estimated horizontal surface velocities:

$$
\begin{aligned}
& \hat{v}_{0 x}=v_{0 x}-\Delta v_{p}-v_{p}(1-\cos \beta \cos \gamma) \\
& \hat{v}_{0 y}=v_{0 y}+v_{p} \cos \beta \sin \gamma+\frac{\left(\Delta v_{z}-v_{0 z}\right)+v_{p} \sin \beta}{\tan \theta_{i} \cos \theta_{s}} .
\end{aligned}
$$

Thus, any bias in the estimated platform velocity translates directly into the along-track component of surface velocity and can be a significant source of error here. In the cross-track direction, the dominant sources of error involve attitude and platform vertical velocity. In particular, vertical velocity errors dominate for small incidence angles where the $\tan \theta_{i}$ term serves to amplify errors. At a platform velocity of $100 \mathrm{~ms}^{-1}$, tolerating biases of $\approx 5 \mathrm{~cm}^{-1}$ in either velocity component implies horizontal velocity accuracy better than $5 \mathrm{~cm}^{-1}$ and attitude accu- racies better than $0.5 \mathrm{mrad}$. The sensitivity to attitude error is a consequence of the aft antenna not tracking directly behind the fore antenna. In this case, a cross-track path length error is incorporated in the interferometric phase measurement.

To investigate the effect of small random errors in platform velocity and attitude on surface velocity estimates, we can reconsider (12) using (17) as the source of phase uncertainty. Assuming the average values of $\beta$ and $\gamma$ are zero, and assuming velocity and attitude errors are independent, the first order result is shown in (19), at the bottom of the page. Fig. 5 shows predicted single-look velocity uncertainties given platform velocity and attitude uncertainties available using current low-cost GPS/INS systems [26]. The solid line shows the squint angle dependence at a constant incidence angle of $45^{\circ}$, while the dotted line shows the incidence angle dependence at a constant squint angle of $45^{\circ}$. The squint angle dependence shows a minimum near $45^{\circ}$, while the incidence angle dependence is monotonically decreasing with increasing incidence angle. At small incidence angles, the uncertainty increases rapidly due largely to vertical velocity variance. This suggests best results are obtained using moderate squint angles and larger incidence angles.

A separate motivation for a large internal angle between fore and aft beams is the possibility to exploit backscattered power measurements to estimate the surface wind direction through wind vector scatterometry. As discussed earlier, knowledge of the wind direction is helpful to account for the component of Doppler velocity due to the phase velocity of Bragg-resonant surface waves. Additional knowledge of the wind speed permits an accounting for surface wind drift, typically 3-5\% of the wind speed at 10-m height. Thus, the DBI and the wind vector scat- 
TABLE I

SIMULATION PARAMETERS

\begin{tabular}{l|l}
\hline Parameter & Specification \\
\hline Frequency & $5.3 \mathrm{GHz}$ \\
Polarization & $\mathrm{VV}$ \\
Bandwith & $5 \mathrm{MHz}$ \\
Pulse width & $10 \mu \mathrm{s}$ \\
Pulse repetition frequency & $12 \mathrm{KHz}$ \\
Transmitted power & $10 \mathrm{~W}$ \\
Receiver noise figure & $3 \mathrm{~dB}$ \\
Platform height & $2000 \mathrm{~m}$ \\
Platform velocity & $100 \mathrm{~m} / \mathrm{s}$ \\
Antenna Baseline (2B) & $1 \mathrm{~m}$ \\
Time lag/integration time & $10 \mathrm{~ms}$ \\
Incidence angles & $35^{\circ}-75^{\circ}$ \\
Squint angle & $30^{\circ}$ \\
Boresight incidence angle & $27.5^{\circ}$ \\
lst Side lobe levels & $20 \mathrm{~dB}$ \\
Azimuth Beamwidth & $7^{\circ}$ \\
Elevation Beamwidth & $30^{\circ}$ \\
Wind velocities & $2-15 \mathrm{~m} / \mathrm{s}$ \\
Wind direction w.r.t. $v_{p}$ & $0-90$ \\
\hline
\end{tabular}

terometer provide complementary measurements of the sea surface.

These design issues suggest reasonably large squint angles in the neighborhood of $45^{\circ}$. There are other design factors that motivate small internal angles between fore and aft looks. For example, smaller squint angles yield a wider cross-track swath, reduce along-track sampling frequency requirements (smaller Doppler shifts), and depending upon the antenna design, reduce sensitivity to polarization mixing effects due to squinting. Thus, several competing factors must be addressed in arriving at a compromise instrument design.

\section{F. Polarization}

Depending upon the choice of antenna architecture, the fore and aft squint of the sensor can result in different polarization characteristics. One design approach is to consider a nominally sidelooking antenna that radiates both fore- and aft-squinted beams, which may be either simultaneous or switched. Another approach is to consider two different antennas physically oriented along the desired squint directions. These may also be power-combined or switched.

While the first approach is attractive, the resulting combination of incidence angle and squint angle yields polarization mixing such that field incident on the ocean surface will consist of a combination of vertical and horizontal polarizations (as- suming a vertically polarized transmitting antenna). To see this, consider an antenna at height $H$ along the $z$-axis above the $x-y$ plane oriented such that the main beam of the antenna lies in the $y-z$ plane (see Fig. 6). The orientation of the propagation vector for incidence angle $\theta$ is

$$
\hat{\mathbf{k}}=\hat{\mathbf{y}} \sin \theta-\hat{\mathbf{z}} \cos \theta
$$

and the orientation of the electric field is

$$
\hat{\mathbf{e}}=\hat{\mathbf{y}} \cos \theta+\hat{\mathbf{z}} \sin \theta .
$$

If the beam is subsequently squinted forward or aft, then the propagation vector $\hat{\mathbf{k}}$ is rotated about the axis of the electric field

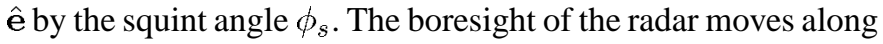
the dotted line in Fig. 6, while the orientation of ê remains the same

$$
\hat{\mathbf{k}}=\hat{\mathbf{x}} \sin \phi_{s}+\hat{\mathbf{y}} \cos \phi_{s} \sin \theta-\hat{\mathbf{z}} \cos \phi_{s} \cos \theta \text {. }
$$

Depending upon the squint and incidence angles, the orientation of $\hat{\mathbf{e}}$ in the plane normal to the squinted $\hat{\mathbf{k}}$ now includes a horizontal component. The horizontal component of ê varies as the vertical component of the magnetic field

$$
\hat{h}_{z}=\hat{\mathbf{z}} \cdot(\hat{\mathbf{k}} \times \hat{\mathbf{e}})=\sin \phi_{s} \cos \theta .
$$

Note that for small incidence angles and large squints, the polarization becomes more horizontal than vertical. Fig. 6 shows the degree of polarization mixing for various squint angles in terms of the percentage of total power contained in the vertical polarization. Polarization mixing is undesirable, especially at large incidence angles where non-Bragg scattering sources impact the horizontally polarized Doppler spectrum. Mixing also complicates interpretation of backscattered power as both polarizations must be considered. For squint angles less than about $30^{\circ}$, however, the fraction of horizontal polarization is reasonably small.

An alternative antenna design approach is to use physically different antennas for fore and aft beams. Rotating an antenna about the $z$-axis causes the boresight to move along the circle in Fig. 6 rather than the line and preserves pure $\mathrm{V}$ or $\mathrm{H}$ polarizations for each beam. The resulting antenna structure, however, may be less conformal to aircraft surfaces.

\section{Airborne DBI fOR COASTAl CURRENT MAPPING}

Having considered a number of measurement issues, in this section, a candidate design for an airborne DBI system is outlined. We consider here a DBI system designed for compatibility with small aircraft. For design purposes, we assume aircraft ve-

$$
\sigma_{v_{0}}^{2}=\frac{2\left(\sigma_{v_{p}}^{2} \sin ^{2} \theta_{i} \sin ^{2} \theta_{s}+\sigma_{\gamma}^{2} v_{p}^{2} \sin ^{2} \theta_{i} \cos ^{2} \theta_{s}+\left(\sigma_{\beta}^{2} v_{p}^{2}+\sigma_{v_{z}}^{2}\right) \cos ^{2} \theta_{i}\right)}{\sin ^{2} 2 \theta_{s} \sin ^{2} \theta_{i}} .
$$




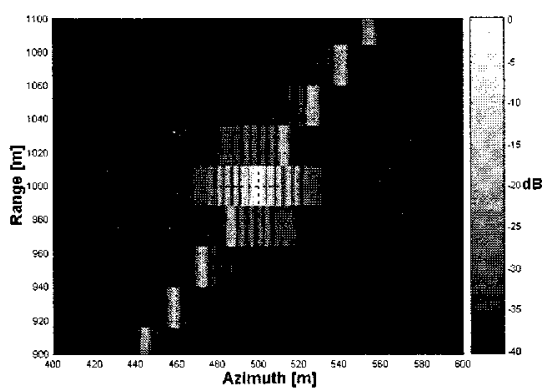

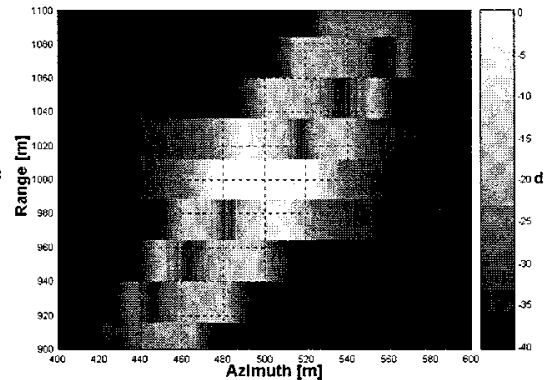

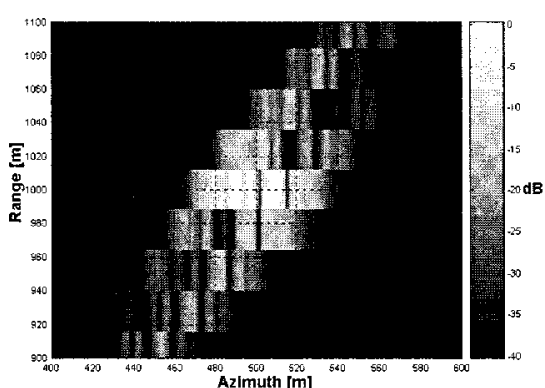

$\mathrm{C}$

Fig. 8. Simulated impulse response for a $30^{\circ}$ squinted SAR at $v_{p}=50 \mathrm{~m} / \mathrm{s}$. (a) Fixed target $T_{i}=200 \mathrm{~ms}$. (b) Fixed target $T_{i}=10 \mathrm{~ms}$. (c) Partially coherent, moving target with phase statistics given by [11, Eq. (1)] and $T_{i}=10 \mathrm{~ms}$.

locity $v_{p} \approx 50-100 \mathrm{~ms}^{-1}$ and aircraft altitude $H \approx 1.5-3 \mathrm{~km}(2$ $\mathrm{km}$, typ). For incidence angles in the range $35^{\circ}-75^{\circ}$, the swath width is typically $6 \mathrm{Km}$. At maximum altitude, unambiguous range considerations limit the PRF to $15.6 \mathrm{KHz}$.

The fundamental choice of operating frequency dictates much of the remaining design of the interferometer. The most widely published ATI-SAR measurements are those of the Jet Propulsion Laboratory's (JPL's) DC-8-based AirSAR system operating primarily at L-band and also at C-band (e.g., [1], [2], [22], [5]). Lower microwave frequencies are sensitive to the longer surface gravity waves, which have longer lifetimes and are more closely associated with the near-surface current. Antenna sizes for these frequencies can become unwieldy for a small aircraft. X-band and higher microwave frequencies are attractive due the higher gain achievable with fixed sized antennas. However, these frequencies are sensitive to the short capillary waves which are themselves largely influenced by local winds. Given these considerations, C-band is a reasonable tradeoff between the desire for sensitivity to somewhat longer gravity-capillary waves and compactness for installation in a small aircraft. Additionally, operation in this band permits intercomparison with other remote sensors including, for example, the ERS-2 and RADARSAT satellites.

For design purposes, a coherence time between 10 and 30 $\mathrm{ms}$ is assumed at C-band. To obtain meaningful interferometric measurements, the delay between two looks at the surface needs to be less than $\tau_{s}$. For nonoverlapping physical apertures, the size of the antenna is limited by the baseline separation. For the case of a single transmitting antenna, the baseline distance is

$$
2 B=2 v_{p} \tau
$$

For $\tau=10 \mathrm{~ms}$ and $v_{p}=50 \mathrm{~ms}^{-1}$, the baseline is $2 B=100$ $\mathrm{cm}$. Allowing for some minimal separation of the apertures, a horizontal aperture size of $60 \mathrm{~cm}$ is reasonable, which yields an approximate half-power beamwidth of $7.5^{\circ}$ for a $30^{\circ}$ squint. The elevation beamwidth is broader to afford coverage over the incidence angles from $35^{\circ}$ to $75^{\circ}$ [Fig. 7(c)].

Since azimuth resolution is limited by the coherence time of the scatterers on the sea surface, it is of questionable value to have a system with extremely fine range resolution. Anticipated operational bandwidth is about $5 \mathrm{MHz}$ providing a range resolution of $30 \mathrm{~m}$ for current applications. The system is designed to be capable of up to $50 \mathrm{MHz}$ bandwidth $(\Delta r=3 \mathrm{~m})$ for other applications. The small fractional bandwidth suggests a microstrip patch array antenna as a good candidate.

To avoid polarization mixing effects and to provide flexibility in the choice of operational squint angles, this DBI system employs separate antennas for each beam (a total of four antennas). These are rectangular microstrip patch array antennas enclosed in an aerodynamic radome. In principle, pairs of fore and aft beams can be combined into a single receiver channel for recording. They can then be separated using Doppler processing. This scheme works provided the PRF of the radar is sufficient to sample both Doppler-shifted echoes simultaneously. It is also desirable that detected power levels are similar in both the fore and aft beams. If they should differ drastically, however, sidelobes from the more energetic beam could interfere or even mask the signal from the weaker beam. For this reason, a switched antenna is preferred. Fig. 7(a) shows the footprint of the fore and aft antenna beams. Fig. 7(b) shows a $\sigma^{\circ}$ contour plot for a constant $5 \mathrm{~ms}^{-1}$ wind speed field. Note the directional modulations of $\sigma^{\circ}$ that translate into different received power levels in the fore and aft beams [Fig. 7(c)] and hence, different SNR. Fig. 7(d) shows a contour plot of the received power level.

Given the squint angles and the aircraft velocity, one can estimate the mean Doppler shift of echoes from the fore and aft beams. These will determine the pulse repetition frequency required of the radar to avoid ambiguities. Mean Doppler shifts are given by

$$
\bar{f}_{d}= \pm \frac{2 v_{p}}{\lambda} \sin \theta_{i} \sin \theta_{s}
$$

For a $30^{\circ}$ squint, $\bar{f}_{d} \leq \pm v_{p} / \lambda$ or approximately $\pm 900 \mathrm{~Hz}$ at $v_{p}=50 \mathrm{~ms}^{-1}$. In addition, the finite antenna beamwidths will impart a spreading about these mean frequencies of approximately $\pm 200 \mathrm{~Hz}$. To avoid aliasing and to allow for variations in airspeed and attitude, one should oversample this considerably. In practice, one can sample much faster than is required while still satisfying unambiguous range constraints. The anticipated minimum along-track sampling frequency (PRF) is approximately $2 \mathrm{kHz}$. 

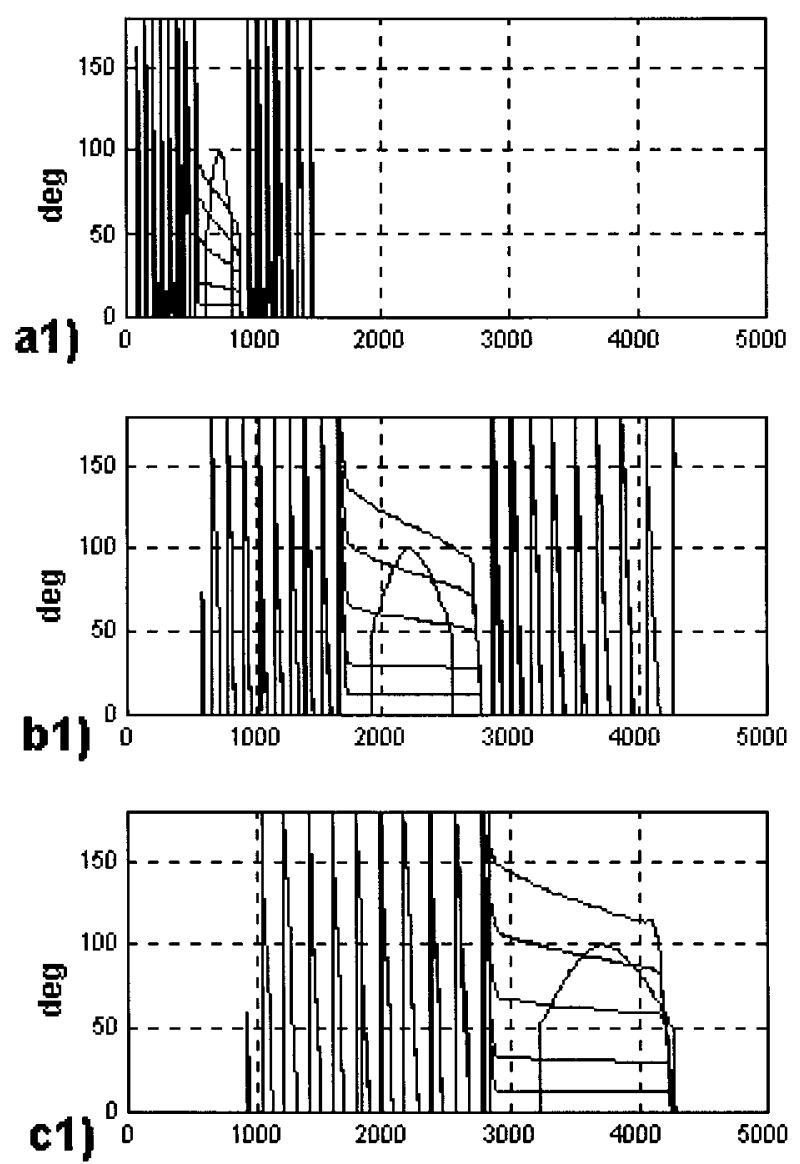
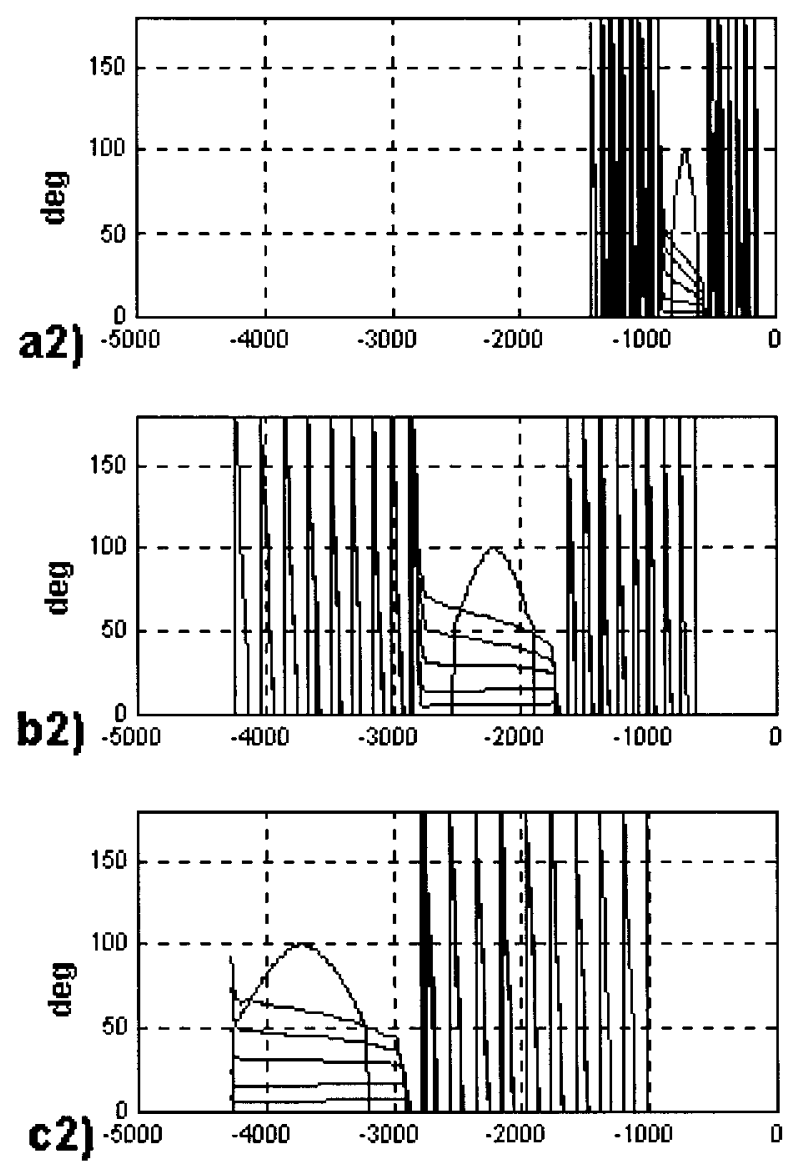

Fig. 9. Phase of $I(x, y)$ at (a) $y=1213 \mathrm{~m}$, (b) $y=3838 \mathrm{~m}$, and (c) $y=6464 \mathrm{~m}$, for the fore (1) and aft (2) beams. Curves from the bottom to the top correspond to responses to uniform velocity fields of $10,25,50,75$, and $100 \mathrm{~cm} / \mathrm{s}$ oriented $60^{\circ}$ from along-track. The normalized physical antenna pattern (truncated to the half-power beamwidth multiplied by 100) is overlaid.

\section{COMPUTER Simulations}

To improve understanding of the system and to assess more accurately its performance, a series of computer simulations have been carried out. Simulations incorporate the design parameters of the airborne instrument and include the effects of platform attitude and velocity errors, directional variation of $\sigma^{\circ}$, finite surface coherence time and its effect on spatial resolution and all operations required in the processing. Simulation parameters are listed in Table I.

First, the impulse response of a squinted SAR to fixed and moving targets has been studied for different integration times. Fig. 8(a) and (b) show the impulse response of a fixed target for $200 \mathrm{~ms}$ and $10 \mathrm{~ms}$ integration time. As expected, the azimuthal resolution is narrower for the longer integration time. Fig. 8(c) shows the impulse response for $10 \mathrm{~ms}$ integration time of a moving, partially coherent target with phase-difference statistics of [11]. Its width is roughly the same as in Fig. 8(b). However, even for this short integration time, decorrelation affects the quality of the focusing.

Fig. 9 shows the phase of $I\left(x, y=y_{0}\right)$ at $y_{0}=1213 \mathrm{~m}, 3838$ $\mathrm{m}$, and $6464 \mathrm{~m}$, computed from (45). As computed, each point in these plots corresponds to the system's response to a pixel located at a given azimuth distance. Since the simulated surface velocity field is constant, the plots may also be viewed as the sequence of interferometric phases obtained from a given pixel as it passes through the fore and aft antenna beams. Constant current fields of $0.1,0.25,0.50,0.75$, and $1.00 \mathrm{~ms}^{-1}$ are considered, which are oriented $60^{\circ}$ from along-track. That is, currents aligned with the direction of the forward squinted beam. In all plots, the projected physical antenna beam (truncated to the half-power beamwidth and multiplied by 100) is indicated.

Fig. 9 shows an interesting result. Since the SAR image for each scatterer appears displaced $x_{\max }=\mathbf{R} \cdot \mathbf{v} / v_{p}$, the higher the scalar product $\mathbf{R} \cdot \mathbf{v}$, the larger the shift of the phase responses from the center of the physical antenna beam. As shown in the appendix and also in [20], the phase response to a moving target is displaced azimuthally in the same manner as is the amplitude response. A bias appears in the averaged phase when the phase jump enters into the half-power beamwidth of the physical antenna (plot c1). This effect does not occur to the same extent for the aft beam (plot c2) since the angle between the velocity field (aligned with the fore beam) and the aft beam is $2 \theta_{s}$. The scalar product is smaller and so the displacement.

Fig. 10 shows velocity biases and standard deviations from simulations including finite SNR. For each simulation, a sequence of interferometric images is produced. Within each sequence, a given surface pixel appears at a slightly different position as it traverses the physical antenna beam. For each position, the incidence angle and the angle relative to the along-track direction 

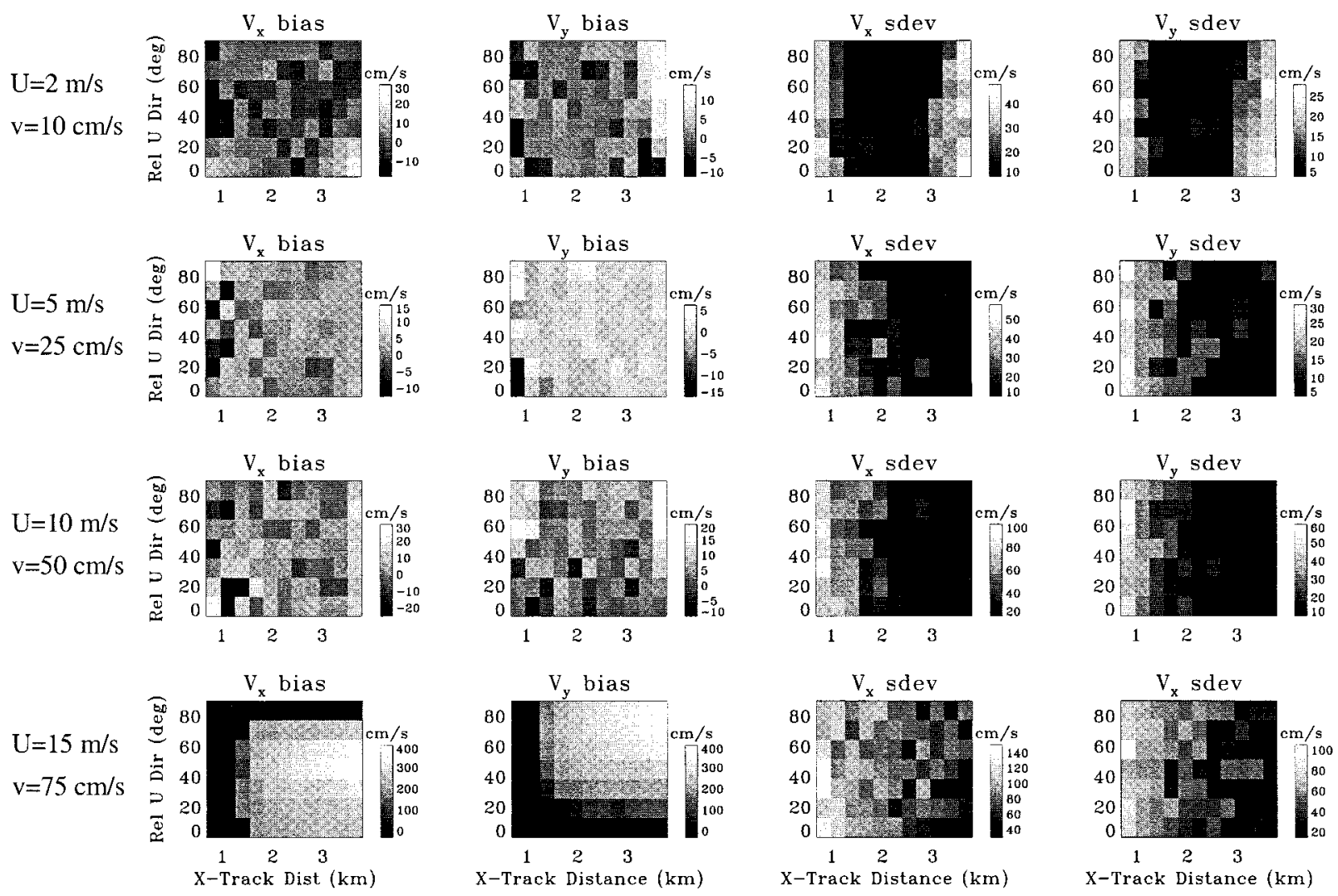

Fig. 10. Measurement biases and standard deviations for four surface velocities: $v=10,25,50$, and $75 \mathrm{~cm} / \mathrm{s}$ (corresponding to winds of $U=2,5,10$, and 15 $\mathrm{m} / \mathrm{s}$ ). In each plot, the abscissa is ground range, and the ordinate is the direction of the surface velocity with respect to along-track direction. Squint angle is fixed at $30^{\circ}$.

are computed. Then (10) is used to derive the radial velocities $\hat{u}_{r}^{ \pm}$ and from them, the velocities in the along-track and cross-track directions are obtained using (11). Finally, from a set of 25 such simulations, means and standard deviations are calculated. These are shown for the along-track and the cross-track directions for four surface velocities $10,25,50$, and $75 \mathrm{~cm} / \mathrm{s}$, which have been associated with winds of $2,5,10$, and $15 \mathrm{~m} / \mathrm{s}$, respectively. NRCS for each case is modeled using the CMOD4 model function.

Abscissas in Fig. 10 indicate distance from the pixel to the ground-track (as in Fig. 7), and ordinates indicate the direction of the surface velocity with respect to along-track. With few exceptions, biases for velocities of $50 \mathrm{~cm} / \mathrm{s}$ and below are distributed about zero. As computed, standard deviations reflect the uncertainty of individual interferometric velocity estimates.

The bias situation is quite different for $u=75 \mathrm{~cm} / \mathrm{s}$, where the biases in both components are much larger than their corresponding standard deviations. As commented, this effect increases with both range and surface velocity, and the maximum occurs when the surface velocity is aligned with the direction of the forward beam. To mitigate this problem, averaging of interferometric phases can be confined to a smaller region within the physical antenna beamwidth. Simulations indicate that for this configuration and wind speeds up to $20 \mathrm{~ms}^{-1}$, negligible bias occurs if averaging is confined to the 1-dB beamwidth of the antenna. This condition guarantees that all the interferometric phase samples in the impulse response lie in the linear region. Of course, this bias reduction comes at the expense of an in- crease in the standard deviation of about $30 \%$, as the effective illumination time $T_{0}$ is reduced.

Computed standard deviations follow basically the shape of the SNR [Fig. 7(c)] with minimum SNR and maximum RMS velocity errors at the swath edges. Note that, with the exception of small incidence angles (small ranges), the relative RMS error (RMSE) decreases with increasing wind speeds since the $\sigma^{\circ}$ increases. This effect compensates the partial loss of correlation between measurements at high winds. Similar results have been simulated for other configurations: altitude, squint angle etc., and could be extrapolated from this particular DBI configuration.

\section{CONCLUSIONS}

This paper has described the principle of operation of an airborne instrument designed to measure sea surface currents in a single pass. It is based on the measurement of two interferograms, one using two antennas pointing forward, the other using two pointing aft. Each provides one radial component of the surface velocity.

To analyze the performance of this instrument, we have reviewed the effects of SNR and finite coherence time of the surface backscatter. The response to the ocean surface of a squinted ATI-SAR has been derived, including platform attitude and velocity errors. An airborne instrument design has been summarized. Finally, system performance has been simulated, showing an inherent limitation in the number of interferometric phase 
samples that can be averaged for particular conditions. This intrinsic limitation arises due to the shift in the position of the SAR response to moving targets. To mitigate it, the number of samples can be reduced by restricting the illumination time to within the $1 \mathrm{~dB}$ beamwidth of the antenna.

\section{APPENDIX \\ SQUINTED ATI-SAR RESPONSE WITH ATTITUDE/VELOCITY ERRORS}

Referring to the geometry of Fig. 1, ideally, the platform is moving at a constant velocity along the $x$-axis, at a height $\mathrm{H}$ from the surface. Taking into account possible errors in the platform movement, the positions of antennas 1 (fore) and 2 (aft) are given by

$$
\begin{aligned}
& \mathbf{A}_{1}=\left(B_{x}, B_{y}, B_{z}+H\right)+\mathbf{v}_{\mathbf{p}} t+\frac{1}{2} \mathbf{a}_{\mathbf{p}} t^{2} \\
& \mathbf{A}_{\mathbf{2}}=\left(-B_{x},-B_{y},-B_{z}+H\right)+\mathbf{v}_{\mathbf{p}} t+\frac{1}{2} \mathbf{a}_{\mathbf{p}} t^{2}
\end{aligned}
$$

where $\mathbf{B}=\left(B_{x}, B_{y}, B_{z}\right)$ is the half-baseline. $\mathbf{B}$ and $\mathbf{v}_{\mathbf{p}}$ can be expressed as

$$
\begin{aligned}
\mathbf{B} & =B(\cos \beta \cos \gamma, \cos \beta \sin \gamma,-\sin \beta) \\
\mathbf{v}_{\mathbf{p}} & =\left(v_{p}+\Delta v_{p}, 0, \Delta v_{z}\right)
\end{aligned}
$$

where $\beta$ and $\gamma$ are the respective pitch and yaw angles. The velocity vector includes error terms in along-track velocity and in vertical velocity to account for possible estimation errors. By definition, the cross-track component of velocity is zero since we have defined a yaw angle for the platform.

The position of a moving observation point (e.g., the sea surface) during a short integration time is approximated by constant velocity and acceleration terms $\mathbf{v}_{\mathbf{0}}$ and $\mathbf{a}_{\mathbf{0}}$

$$
\mathbf{P}=\left(x_{0}, y_{0}, z_{0}\right)+\mathbf{v}_{\mathbf{0}} t+\frac{1}{2} \mathbf{a}_{\mathbf{0}} t^{2}
$$

Computation of the squinted ATI-SAR response including attitude and velocity errors generalizes the results described in [13], [20] for the sidelooking ideal case. The echo signals received by the ATI-SAR are proportional to

$$
S_{r}^{1,2}(\mathbf{P}, t)=r(\mathbf{P}, t) \exp \left(-j k R^{ \pm}(\mathbf{P}, t)\right)
$$

where $r(\mathbf{P}, t)$ is the reflection coefficient of the surface at the point $\mathbf{P}$ and time $t$. Assuming that antenna 2 transmits, and both antennas receive, the two-way distance from antenna 2 to the target $\left(R^{+}=2\left|\mathbf{P}-\mathbf{A}_{\mathbf{2}}\right|\right)$ and the distance from antenna 2 to the target and back to antenna $1\left(R^{-}=\left|\mathbf{P}-\mathbf{A}_{1}\right|+\left|\mathbf{P}-\mathbf{A}_{\mathbf{2}}\right|\right)$ are approximately given by

$$
\begin{aligned}
& R^{+}(t) \approx \\
& 2 R\left[1+\frac{z_{0}^{2}+B^{2}+2\left(x_{0} B_{x}+y_{0} B_{y}+z_{0} B_{z}-z_{0} H-B_{z} H\right)}{2 R^{2}}\right.
\end{aligned}
$$

$$
\begin{aligned}
& -\frac{\left(x_{0}+B_{x}\right) v_{x}+\left(y_{0}+B_{y}\right) v_{y}+\left(z_{0}+B_{z}-H\right) v_{z}}{R^{2}} t \\
& \left.+\frac{v^{2}-\left(x_{0}+B_{x}\right) a_{x}-\left(y_{0}+B_{y}\right) a_{y}-\left(z_{0}+B_{z}-H\right) a_{z}}{2 R^{2}} t^{2}\right]
\end{aligned}
$$

$$
\begin{aligned}
& R^{-}(t) \approx \\
& 2 R\left[1+\frac{z_{0}^{2}+B^{2}-2 z_{0} H}{2 R^{2}}-\frac{x_{0} v_{x}+y_{0} v_{y}+\left(z_{0}-H\right) v_{z}}{R^{2}} t\right. \\
& \left.+\frac{v^{2}-x_{0} a_{x}-y_{0} a_{y}-\left(z_{0}-H\right) a_{z}}{2 R^{2}} t^{2}\right]
\end{aligned}
$$

where $R^{2}=x_{0}^{2}+y_{0}^{2}+H^{2}$, and $B^{2}, v^{2}$, and $a^{2}$ are all inner products of corresponding vector quantities. Here we have defined the relative velocity $\mathbf{v}=\left(v_{x}, v_{y}, v_{z}\right)=\mathbf{v}_{\mathbf{p}}-\mathbf{v}_{\mathbf{0}}$ and acceleration $\mathbf{a}=\left(a_{x}, a_{y}, a_{z}\right)=\mathbf{a}_{\mathbf{p}}-\mathbf{a}_{\mathbf{0}}$. The signals collected by antennas 1 and 2 are given by

$$
\begin{aligned}
S_{0}^{1,2}(x, y, t)= & \iint S_{r}^{1,2}\left(x_{0}, y_{0}, t\right) G_{x}\left(x_{0}, t\right) G_{y}\left(y_{0}\right) \delta \\
& \cdot\left(y-y_{0}+\left(x-x_{0}\right) \tan \theta_{s}\right) d x_{0} d y_{0}
\end{aligned}
$$

where $G_{x}\left(x_{0}, t\right)=\exp \left(-2\left(x_{0}-\bar{x}-v_{x} t+B\right)^{2} / X^{2}\right)$ is the squinted antenna pattern projected into the along-track direction. The antenna pattern maximum is located at $x=\bar{x}$, and the width of the antenna footprint in the along-track direction is $X . G_{y}\left(y_{0}\right)$ is the antenna pattern projected in the cross-track direction, so that the composite antenna pattern is given by the product $G_{x} G_{y}$. This decomposition neglects the effect of the differential range between the leading and trailing edges of the cross-track antenna pattern and so is valid for modest squints or narrow beams only.

The signals collected by antennas 1 and 2 are then cross-correlated in the processor with reference signals

$$
\begin{aligned}
& h_{1}(x, R, t)=\exp \left(j \frac{2 k}{R}\left[-(x+B) v_{p} t+\frac{v_{p}^{2}}{2} t^{2}\right]\right) \\
& h_{2}(x, R, t)=\exp \left(j \frac{2 k}{R}\left[-x v_{p} t+\frac{v_{p}^{2}}{2} t^{2}\right]\right)
\end{aligned}
$$

leading to a pair of complex images, $i_{1,2}(x, y)$. The complex ATI SAR image is then formed by taking the ensemble average product

$$
\begin{aligned}
I(x, y)= & \left\langle i_{1}^{*}(x, y) i_{2}(x, y)\right\rangle \\
= & \iiint \iint\left\langle r^{*}\left(x_{1}, y_{1}, t_{1}\right) r\left(x_{2}, y_{2}, y_{2}\right) \exp (j k \Delta R)\right\rangle \\
& \cdot G_{x}\left(x_{1}, t_{1}\right) G_{y}\left(y_{1}\right) G_{x}\left(x_{2}, t_{2}\right) G_{y}\left(y_{2}\right) \\
& \cdot h_{1}^{*}\left(x_{1}, R_{1}, t_{1}\right) h_{2}\left(x_{2}, R_{2}, t_{2}\right) \\
& \cdot d x_{1} d x_{2} d y_{1} d y_{2} d t_{1} d t_{2} .
\end{aligned}
$$


Consistent with other developments, the term within the expectation is modeled assuming spatially uncorrelated backscatter and a Gaussian temporal correlation function

$$
\begin{aligned}
& \left\langle r^{*}\left(x_{1}, y_{1}, t_{1}\right) r\left(x_{2}, y_{2}, t_{2}\right) e^{j k \Delta R}\right\rangle \\
& \quad=\sigma^{\circ}\left(x_{1}, y_{1}, T\right) \delta\left(x_{1}-x_{2}, y_{1}-y_{2}\right) \exp \left(-\tau^{2} / \tau_{s}^{2}\right) e^{j k \Delta R}
\end{aligned}
$$

where $\tau=t_{1}-t_{2}$ and $T=\left(t_{1}+t_{2}\right) / 2$. With this assumption, the differential range term in the complex exponential becomes

$$
\begin{aligned}
& \Delta R=R^{+}\left(x_{1}, y_{1}, t_{1}\right)-R^{-}\left(x_{1}, y_{1}, t_{2}\right) \\
& \approx \frac{2}{R}\left[\left(x_{1} B_{x}+y_{1} B_{y}+\left(z_{1}-H\right) B_{z}\right)\right. \\
& -\left(x_{1} v_{x}+y_{1} v_{y}+\left(z_{1}-H\right) v_{z}\right)\left(t_{1}-t_{2}\right) \\
& -\left(B_{x} v_{x}+B_{y} v_{y}+B_{z} v_{z}\right) t_{1} \\
& +\left(v^{2}-x_{1} a_{x}-y_{1} a_{y}-\left(z_{1}-H\right) a_{z}\right) \frac{t_{1}^{2}-t_{2}^{2}}{2} \\
& \left.-\left(B_{x} a_{x}+B_{y} a_{y}+B_{z} a_{z}\right) \frac{t_{1}^{2}}{2}\right]
\end{aligned}
$$

which can be written in a more compact form as

$$
\begin{array}{r}
\Delta R \approx \frac{2}{R}\left[\mathbf{R} \cdot \mathbf{B}-(\mathbf{R} \cdot \mathbf{v}) \tau-(\mathbf{B} \cdot \mathbf{v})\left(T+\frac{\tau}{2}\right)\right. \\
\left.+\left(v^{2}-\mathbf{R} \cdot \mathbf{a}\right) \tau T-\frac{\mathbf{B} \cdot \mathbf{a}}{2}\left(T+\frac{\tau}{2}\right)^{2}\right]
\end{array}
$$

where $\mathbf{R}=\left(x_{0}, y_{0}, z_{0}-H\right)$ is now the vector from the point on the surface to the midpoint of the interferometer. Since the coherence time is a function of the electromagnetic wavelength, the sea state and also the spatial resolution, which in turn, depends on the integration time and coherence time, it is proper to retain it inside the integral. However, for moderate resolutions, the coherence time's dependence on spatial resolution is small and can be assumed to be constant for a particular wind speed and electromagnetic wavelength.

Inserting (35), (36), (38), and (40) into (37), renaming the variables $\left(x_{1}, y_{1}\right)$ to $\left(x_{0}, y_{0}\right)$, we obtain

$$
\begin{aligned}
& I(x, y) \ln \left(\iint \sigma^{\circ}\left(x_{0}, y_{0}, T\right) \exp \left(-\tau^{2} / \tau_{s}^{2}\right) G_{y}^{2}\left(y_{0}\right)\right. \\
& =\quad \exp \left(-\frac{2}{X^{2}}\left[2\left(x_{0}-\bar{x}\right)^{2}+v_{x}^{2}\left(2 T^{2}+\tau^{2} / 2\right)\right.\right. \\
& \left.\left.+2 B^{2}-4 v_{x} T\left(x_{0}-\bar{x}\right)-2 B v_{x} \tau\right]\right) \\
& \quad \cdot \exp \left(-j \frac{2 k}{R}\left[x\left(B-v_{p} \tau\right)-B v_{p}(T+\tau / 2)+v_{p}^{2} T \tau\right]\right) \\
& \cdot \exp (j k \Delta R) \delta\left(y-y_{0}+\left(x-x_{0}\right) \tan \theta_{s}\right) d \tau d T d x_{0} d y_{0}
\end{aligned}
$$

and integrating over $y_{0}$, the complex ATI-SAR image is expressed

$$
\begin{aligned}
& I(x, y) \\
& =\iiint \sigma^{\circ}\left(x_{0}, y+\left(x-x_{0}\right) \tan \theta_{s}, T\right) \exp \left(-\tau^{2} / \tau_{s}^{2}\right) \\
& \quad \cdot G_{y}^{2}\left(y+(x-x 0) \tan \theta_{s}\right) \\
& \quad \cdot \exp \left(-\frac{2}{X^{2}}\left[2\left(x_{0}-\bar{x}\right)^{2}+v_{x}^{2}\left(2 T^{2}+\tau^{2} / 2\right)\right.\right. \\
& \left.\left.\quad+2 B^{2}-4 v_{x} T\left(x_{0}-\bar{x}\right)-2 B v_{x} \tau\right]\right) \\
& \quad \cdot \exp \left(-j \frac{2 k}{R}\left[x\left(B-v_{p} \tau\right)-B v_{p}(T+\tau / 2)+v_{p}^{2} T \tau\right]\right) \\
& \quad \cdot \exp (j k \Delta R) d \tau d T d x_{0} .
\end{aligned}
$$

It is known that the azimuth resolution is limited by an effective integration time given by (5). Consequently, nearly optimum spatial resolution is achieved for integration times larger than but of similar order to $\tau_{s}$, that is, $\tau_{s} \leq T_{i} \ll X / v_{p}$. The use of short integration times permits more independent interferometric phase measurements from which radial velocity is estimated. We desire many independent estimates to reduce the variance of the estimated velocity. If the integration time is of the order of the coherence time, then the following approximation also holds:

$$
k \mathbf{B} \cdot \mathbf{a} T_{i}^{2} \ll R
$$

Using this approximation and expanding the $\Delta R$ term in (42), we obtain

$$
\begin{aligned}
I(x, y) & =\sqrt{\pi} \exp \left(-\frac{4 B^{2}}{X^{2}}\right) T_{a} \iint \sigma^{\circ}\left(x_{0}, y+\left(x-x_{0}\right) \tan \theta_{s}, T\right) \\
\cdot & G_{y}^{2}\left(y+\left(x-x_{0}\right) \tan \theta_{s}\right) \\
\cdot & \exp \left(\frac{-4\left(x_{0}-\bar{x}-v_{x} T\right)^{2}}{X^{2}}\right) \\
\cdot & \exp \left(-j \frac{2 k B}{R}\left[x-\frac{\mathbf{R} \cdot \mathbf{B}}{B}\right]\right) \\
\cdot & \exp \left(j \frac{2 k}{R}\left[B v_{p}\left(1-\frac{\mathbf{B} \cdot \mathbf{v}}{B v_{p}}\right) T-\frac{\mathbf{B} \cdot \mathbf{a}}{2} T^{2}\right]\right) \\
\cdot & \exp \left(\left\{j \frac{k v_{p}}{R}\left[\left(x-\frac{\mathbf{R} \cdot \mathbf{v}}{v_{p}}\right)+\frac{v^{2}-v_{p}^{2}-\mathbf{R} \cdot \mathbf{a}}{v_{p}} T\right]\right.\right. \\
& \left.\left.+\frac{2 B}{X^{2}} v_{x}\right\}^{2} T_{a}^{2}\right) d T d x_{0} .
\end{aligned}
$$

Finally, the squinted ATI-SAR response is obtained by integrating over $T$ in the interval $\left[-T_{i} / 2, T_{i} / 2\right]$ yielding

$$
\begin{aligned}
& I(x, y) \\
& \quad=\sqrt{\pi} T_{i} \int T_{a} \sigma^{\circ}\left(x_{0}, y+\left(x-x_{0}\right) \tan \theta_{s}, 0\right)
\end{aligned}
$$




$$
\begin{aligned}
& \cdot G_{y}^{2}\left(y+\left(x-x_{0}\right) \tan \theta_{s}\right) \\
& \cdot \exp \left(-\frac{4 B^{2}}{X^{2}}\left[1-\frac{v_{x}^{2} T_{a}^{2}}{X^{2}}\right]\right) \\
& \cdot \exp \left(-\frac{k}{R}\left[\frac{k T_{a}^{2}}{R}\left(v^{2}-v_{p}^{2}-\mathbf{R} \cdot \mathbf{a}\right)^{2}+j \mathbf{B} \cdot \mathbf{a}\right] \frac{T_{i}^{2}}{12}\right) \\
& \cdot \exp \left(-\frac{4\left(x_{0}-\bar{x}\right)^{2}}{X^{2}}\right) \\
& \cdot \exp \left(-\frac{k^{2} T_{a}^{2} v_{p}^{2}}{R^{2}}\left[x-\frac{\mathbf{R} \cdot \mathbf{v}}{v_{p}}\right]^{2}\right) \\
& \cdot \exp \left(j \frac{4 k B v_{x} v_{p} T_{a}^{2}}{R X^{2}}\left[x-\frac{\mathbf{R} \cdot \mathbf{v}}{v_{p}}\right]\right) \\
& \cdot \exp \left(-j \frac{2 k B}{R}\left[x-\frac{\mathbf{R} \cdot \mathbf{B}}{B}\right]\right) d x_{0} .
\end{aligned}
$$

The various exponential terms in this expression can now be interpreted. Given the assumptions made to get to this point, the first two exponential terms are very nearly equal to unity. The third exponential represents the modulation of the echo by the real-aperture antenna beam. The fourth exponential term is the SAR amplitude response, indicating a maximum at $x_{\max }=$ $\mathbf{R} \cdot \mathbf{v} / v_{p}$. This is the azimuthal translation of the SAR amplitude image due to radial motion or the velocity bunching term commonly associated with SAR imaging of the ocean. The fifth exponential is a velocity bunching term for the phase, as discussed in [20]. Note that it passes through zero phase at the SAR image maximum $x_{\max }$. Thus, the phase and amplitude responses are similarly shifted in azimuth due to radial scatterer motion. The final exponential term is the desired interferometric phase. When evaluated at $x=x_{\max }$, the phase of this term is given by (8).

\section{ACKNOWLEDGMENT}

The authors gratefully acknowledge helpful discussions regarding squinted SAR with M. Bara, Universitat Politecnica de Catalunya, Barcelona, Spain, and the comments of the anonymous reviewers.

\section{REFERENCES}

[1] R. M. Goldstein, T. P. Barnett, and H. A. Zebker, "Remote sensing of ocean currents," Science, vol. 246, pp. 1282-1285, 1989.

[2] M. Marom, L. Shemer, and E. B. Thornton, "Energy density directional spectra of a nearshore wave field measured by interferometric synthetic aperture radar," J. Geophys. Res., vol. 96, no. C12, pp. 22 125-22 134, 1991.

[3] D. R. Thompson and J. R. Jensen, "Synthetic aperture radar interferometry applied to ship-generated internal waves in the 1989 Loch Linnhe experiment," J. Geophys. Res., vol. 98, no. C6, pp. 10 259-10 269, 1993.

[4] G. O. Marmorino, D. R. Thompson, H. C. Graber, and C. L. Trump, "Correlation of oceanographic signatures appearing in synthetic aperture radar and interferometric synthetic aperture radar imagery with in situ measurements," J. Geophys. Res., vol. 102, no. C8, pp. 18 723-18 736, 1997.

[5] H. C. Graber, D. R. Thompson, and R. E. Carande, "Ocean surface features and currents measured with synthetic aperture radar inteferometry and hf radar," J. Geophys. Res., vol. 101, no. C11, pp. 25 813-25,832, 1996.

[6] E. Rodriguez, D. Imel, and B. Houshmand, "Two-dimensional surface currents using vector along-track interferometry," in Proc. PIERS'95, Seattle, WA, 1995, p. 763.
[7] W. J. Plant, E. A. Terray, R. A. Pettit Jr., and W. C. Keller, "The dependence of microwave backscatter from the sea on illuminated area, correlation times and lengths," J. Geophys. Res., vol. 99, no. C5, pp. 9705-9723, 1994.

[8] F. C. Jackson and D. R. Lyzenga, "Microwave techniques for measuring directional wave spectra," in Surface Waves and Fluxes, G. L. Geernhaert and W. J. Plant, Eds. Amsterdam, The Netherlands: Kluwer, 1990, vol. 2, ch. 14, pp. 221-264.

[9] W. J. Pierson and L. Moskowitz, "A proposed spectral form for fully developed seas based on the similarity theory of S. A. Kitaigorodskii," J. Geophys. Res., vol. 69, pp. 5181-5203, 1964.

[10] A. K. Fung and K. K. Lee, "A semi-empirical sea spectrum model for scattering coefficient estimation," IEEE J. Oceanic Eng., vol. OE-7, pp. $166-176,1982$

[11] R. D. Chapman, B. L. Gotwols, and R. E. Sterner II, "On the statistics of the phase of microwave backscatter from the ocean surface," J. Geophys. Res., vol. 99, no. C8, pp. 16293-16301, 1994.

[12] W. R. Alpers and C. Bruening, "On the relative importance of motionrelated contributions to the sar imaging mechanism of ocean surface waves," IEEE Trans. Geosci. Remote Sensing, vol. GE-24, pp. 873-885, Nov. 1986.

[13] M. Bao, C. Bruning, and W. Alpers, "Simulation of ocean waves imaging by an along-track interferometric synthetic aperture radar," IEEE Trans. Geosci. Remote Sensing, vol. 35, pp. 618-631, May 1997.

[14] K. C. Ewans, "Observations of the directional spectrum of fetch-limited waves," J. Phys. Oceanogr., vol. 28, no. 3, pp. 495-512, 1998.

[15] D. R. Lyzenga, "Interaction of short surface and electromagnetic waves with ocean fronts," J. Geophys. Res., vol. 96, no. C6, pp. 10 765-10 772, 1991.

[16] E. M. Poulter, M. J. Smith, and J. A. McGregor, "Microwave backscatter from the sea surface: Bragg scattering by short gravity waves," J. Geophys. Res., vol. 99, no. C4, pp. 7,929-7,944, 1994.

[17] D. Moller, S. J. Frasier, D. L. Porter, and R. E. McIntosh, "Radar-derived interferometric surface currents and their relation to subsurface current structure," J. Geophys. Res., vol. 103, no. C6, pp. 12 839-12 852, 1998.

[18] X. Zhang, "Wavenumber spectrum of very short wind waves: An application of two-dimensional slepian windows to spectral estimation," $J$. Atmos. Ocean. Tech., vol. 11, no. 2, pp. 489-505, 1994.

[19] W. J. Plant, "The modulation transfer function: Concept and applications," in Radar Scattering From Wind Waves, K. J. Komen and W. A. Oost, Eds: Kluwer, 1989, pp. 155-172.

[20] M. Bao, W. Alpers, and C. Bruning, "A new nonlinear integral transform relating ocean wave spectra to phase image spectra of an along-track interferometric synthetic aperture radar," IEEE Trans. Geosci. Remote Sensing, vol. 37, pp. 461-466, Jan. 1999.

[21] L. Shemer and E. Kit, "Simulation of an interferometric synthetic aperture radar imagery of an ocean system consisting of a current and a monochromatic wave," J. Geophys. Res., vol. 96, no. C12, pp. $22063-22073,1991$

[22] R. E. Carande, "Estimating ocean coherence time using dual-baseline interferometric synthetic aperture radar," IEEE Trans. Geosci. Remote Sensing, vol. 32, July 1994.

[23] K. S. Miller and M. M. Rochwarger, "A covariance approach to spectral moment estimation," IEEE Trans. Inform. Theory, vol. IT-18, no. 5, pp. 588-596, 1972.

[24] E. Rodriguez and J. M. Martin, "Theory and design of interferometric synthetic aperture radars," Proc. Inst. Elect. Eng. F, vol. 139, no. 2, 1992.

[25] H. A. Zebker and J. Villasenor, "Decorrelation in interferometric radar echoes," IEEE Trans. Geosci. Remote Sensing, vol. 30, pp. 950-959, Sept. 1992.

[26] "Miniature integrated GPS/INS system, C-MIGITSTM II: Preliminary information sheet, Rev. 04-22-1997," Boeing Corp., Seattle, WA, Apr. 1997.

Stephen J. Frasier (S'93-M'95), photograph and biography not available at the time of publication.

Adriano J. Camps (S'91-A'97), photograph and biography not available at the time of publication. 